\title{
Analytical bi-level multi-local-world complex network model on fresh agricultural products supply chain
}

\author{
Yunqing Liu ${ }^{1,3}$, Shiwei $\mathrm{Xu}^{1,2,4^{*}}$, J iajia Liu ${ }^{1,2,4}$, J iayu Zhuang ${ }^{1,2,4}$ \\ (1. Agricultural Information Institute of Chinese Academy of Agricultural Sciences, Beijing 100081, China; \\ 2. Key Laboratory of Agricultural Information Service Technology, Ministry of Agriculture and Rural Affairs, Beijing 100081, China; \\ 3. Institute of Environment and Sustainable Development in Agriculture, Chinese Academy of Agriculture Science, Beijing 100081, \\ China; 4. Research Center of Agricultural Monitoring and Early Warning Engineering Technology, Beijing 100081, China)
}

\begin{abstract}
Lately, in some regions and seasons in China, urban consumers have paid high in buying fresh agricultural products while farmers get unreasonable income from producing them. To seek the reason for the phenomenon and explore ways to simulate it, this study constructed and implemented a complex network model named the Bi-Level Multi-Local-World (BI-MLW model) with characteristics of an interdependent coupling relationship between its participants. To verify the validity of the model, this study implemented an experimental simulation under Small Decentralized Operation Mode (SDOM) and Large Centralized Operation Mode (LCOM) scenarios using Cucurbita pepo and Cucumber in the Tianjin area of China as sample empirical products. Results indicate that nodes do not increase edges rapidly which reflects that even large firms in agricultural business cannot occupy markets fleetly. Furthermore, under the SDOM scenario the BI-MLW model exposes scale-free features with a small average degree value and low average clustering coefficient, while under the LCOM scenario, the model displays a rising average clustering coefficient and a lowered average path length. Both of which are consistent with the common view in literature and features of reality. Thus, the BI-MLW model specially designed for fresh agricultural products supply chain can improve the descriptive ability than conventional Erdös-Rényi (ER), Barabási-Albert (BA), Bianconi-Barabási (BB) network models.
\end{abstract}

Keywords: fresh agricultural products, supplying process, supply chain, complex network, multi-local-world model DOI: $10.25165 /$ j.jiabe.20221501.6353

Citation: Liu Y Q, Xu S W, Liu J J, Zhuang J Y. Analytical bi-level multi-local-world complex network model on fresh agricultural products supply chain. Int J Agric \& Biol Eng, 2022; 15(1): 208-215.

\section{Introduction}

Many complex systems are directly or indirectly related to people, where everyone influences the functioning of the network but not anyone controls the global behavior of the whole system, this is even truer due to the enormous number of participants in the agricultural supplying process.

With the rapid development of modern Internet and logistics, the supply "chain" has gradually evolved into a "network structure". The behavior dependence and coupling between the participants become increasingly close, and the characteristics of cooperative symbiosis and mutually beneficial coexistence become more and more obvious. Farmers cannot perceive their state in the whole supplying system, so they cannot adjust their production scale and growth path to fit into the market, they also cannot find the most suitable traders to raise their bargaining \& risk resistance ability effectively. Moreover, it is also impossible for middleman \& wholesalers to explore the most profitable upstream \& downstream dealers. In short, inspect the dynamics of the supplying system

Received date: $2020-12-13 \quad$ Accepted date: $2021-11-18$

Biographies: Yunqing Liu, $\mathrm{PhD}$ candidate, Assistant Researcher, research interest: early warning network modeling. Email: liuyunqing@caas.cn; Jiayu Zhuang, MS, Associate Researcher, research interest: agricultural information analysis, Email: zhuangjiayu@caas.cn; Jiajia Liu, MS, Assistant Researcher, research interest: agricultural information analysis, Email: liujiajia@caas.cn.

*Corresponding author: Shiwei $\mathrm{Xu}, \mathrm{PhD}$, Researcher, research interest: agricultural information monitoring and early warning. No.12 Zhongguancun South Street, Haidian District, Beijing 100081, China. Tel: +86-10-82109902, Email: xushiwei@caas.cn. from an overall point of view, improve the upward trading efficiency, and stabilize the supply system are in need imperatively.

Complex Network theory abstracts objects and their connections into nodes and edges (connections among them), making use of quantitative metrics to depict the internal interaction and mutual relationship. It can also capture the dynamic structure of the system ${ }^{[1,2]}$, describe behaviors of evolvements of the network as a whole ${ }^{[3,4]}$, which has been widely applied in mathematics ${ }^{[5]}$, physics $^{[6]}$, computer $^{[7]}$, biology ${ }^{[8]}$, economics ${ }^{[9]}$, sociology ${ }^{[10,11]}$, and other fields.

In the early studies of complex networks, Erdös and Rényi use the stochastic graph Model (Erdös-Rényi-model, ER-Model) ${ }^{[12]}$ to describe the relationship between nodes. Randomly select edges between any two nodes with equal probability. However, Barabási and Albert ${ }^{[13]}$ found that connection probability is not equal, more than $80 \%$ of the nodes have fewer than 4 links, while very few nodes have more than 1000 links. The distribution of the number of links follows the "power law distribution", so they add "Network Growth" and "Preferential Attachment" mechanism in Barabási-Albert model (BA model) ${ }^{[13]}$, thus resulting in the characteristic of scale-free. However, empirical analysis of the World Trade Web (WTW ${ }^{[14,15]}$ found that a node is not selected among all the nodes in the World, global preference connection mechanism does not conform to the real situation. Then Bianconi and Barabási proposed Bianconi-Barabási model (BB model) ${ }^{[16]}$, which takes into account the fitness of nodes in the growth mechanism, and the fitness and degree jointly determine the probability of connecting existing nodes in the network with newly added nodes. Caldarelli et al. ${ }^{[17]}$ showed that regardless of the 
fitness distribution, the network generated according to the fitness model has the scale-free feature. Further empirical research shows that the number of edges of nodes not only depends on the degree and fitness of existing nodes but also relates to the category of new nodes. Therefore, Chen ${ }^{[18,19]}$ and other scholars suggested the concept of Multi-local-world model (MLW), the basic idea is to replace the global priority connection mechanism in BA model with a local world priority connection mechanism ${ }^{[20]}$, divide the growth mechanism into the growth of the local world, growth of node, and growth of edges. On this basis, researchers have further extended and deepened the construction. However, literature research shows that related complex network model has not been widely used in the field of agriculture.

So far, due to geographical location, information asymmetry, and many other factors, participants in the agricultural supply chain can only choose partners within a certain range of scope (hereinafter referred to as "local world"), rather than in the entire network. Moreover, fresh agricultural products, with more vulnerable storage and limited inventory time, are badly in need of a more descriptive model.

Therefore, based on the classical Multi-Local-World model, a Bi-Level Multi-Local-World model for fresh agriculture product supply chain (hereinafter referred to as BI-MLW model) was constructed, and empirical analysis was carried out based on the model.

Supply Chain Network $(\mathrm{SCN})^{[6]}$ and Supply Chain System (SCS) in this study all refer to a relationship between participating subjects, rather than "Internet" or "E-commerce".

\section{Materials and methods}

As mentioned above, the construction of a complex network model is the process of abstracting objects as nodes, implementing their relations as edges through an analytical mechanism, like establishment, retention, and disconnection of the network, then "generate" and analyze the network by means of computer simulation.

\subsection{Model description and hypothesis}

This study assumes and restricts research objects to supply chain participants, leaving aside other factors that influence it. Depart from the focal node's view, the study analyzes the supply network as a whole, in addition, state the following hypothesis (Figure 1):

1) Nodes represents different categories of participants, including farmers (household peasant, cooperative producers collectively referred to as farmers), assemblers (middleman assemble agricultural products from producers collectively referred to as assemblers), processors and wholesalers (collectively referred to as wholesalers), retailers, denoted by $w_{f}, w_{a}, w_{w}$, and $w_{r}$, respectively. In addition, this study only involves the above four types of participants, excluding prenatal raw material suppliers and other participants.

2) Edges represent the interaction between nodes, such as the flow of products, formal (or informal) information, or the presence of capital exchange among them, online interaction is considered to set up the network edge ${ }^{[21]}$. The strength of the interaction is represented by the weights of edges.

3) Multi-Local-World (MLW) represents the region where the node is located and its optional range. In the actual supply chain network, limited by geographical location, consumption preference, and other factors, when adding new nodes to the network, partners cannot be selected from all existing nodes, and the addition of new nodes has little impact on nodes in other regions, which in turn are mainly affected by nodes in the regional network. For example, farmers with a small production scale can only trade with a part of assemblers in the region and are influenced by their pricing policies and acquisition rules, while internet live broadcasting can sell products within a larger circle of friends.

4) Level of the local World represents the Level of the optional range. The first level of the local world depends on the category of the node itself and the proportion of various nodes in the network. In other words, the category of the node itself determines the transaction mode of its first layer of the local environment. The second level depends on three factors as the current state of the node, the current position of the local environment, and the current remaining freshness. This study further assumes that nodes can also be connected across hierarchies and local environments, thus providing the possibility of establishing long-term connections across hierarchies.

5) There are no repeated connections and self-circulation (trading with oneself). These factors are not considered in the model. Any kind of strategic behavior of firms is not included either.

6) Dealings are roughly divided into two scenarios: a) Small Decentralized Operation Mode (SDOM). In this mode, most farmers barely develop new channels, buyers, wholesalers, and retailers transport products in a certain downstream path to the final customer. Deals can only be done by the established marketing channel, where each firm trades exclusively with partners located in the previous or next level. The characteristics of most nodes are around a certain mean value, which conforms to the characteristics of normal distribution. b) Large Centralized Operation Mode (LCOM). With the rapid development of modern Internet and logistics, farmers, consumers, wholesalers, retailers and service providers, etc., no longer deal only with local firms but relate to each other freely, representing a typical power-law feature ${ }^{[22]}$, which has been verified in industrial supply chain network.

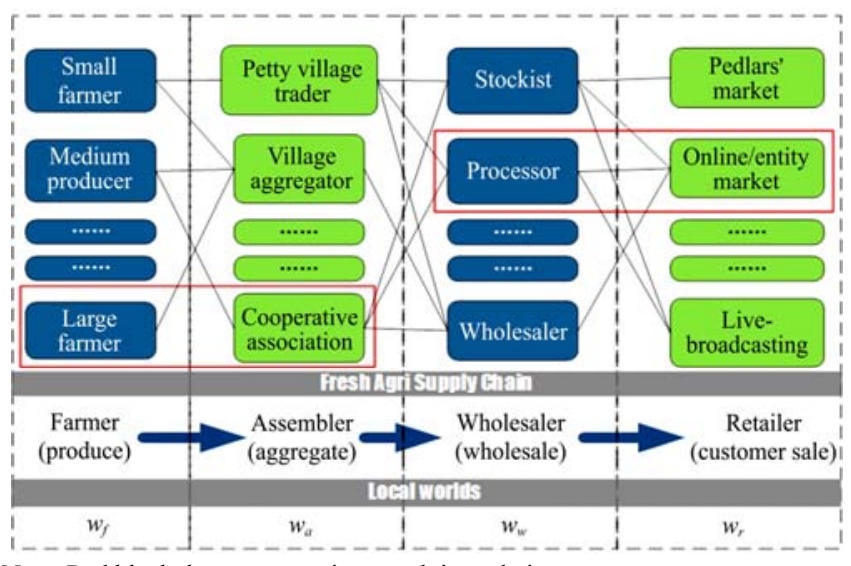

Note: Red block denotes emerging supplying relations.

Figure 1 Description of agri-supply chain and multi-local-world

\subsection{Edge connection mechanism}

Under the model setting of a two-level local environment, adding a new node should go through two basic steps: selecting the first-layer connection environment and selecting the second-layer connection environment within the selected first-layer connection environment. The connection mechanism is as follows:

1) First Level local world

Under the SDOM and LCOM scenarios, the trading probability in the first-level local world is listed in Table 1. 
Table 1 Table of connection probability first level

\begin{tabular}{cccccc}
\hline \multirow{2}{*}{ Mode } & Local-world & $w_{f}$ & $w_{a}$ & $w_{w}$ & $w_{r}$ \\
\hline \multirow{3}{*}{ SDOM } & $w_{f}$ & $P_{f f}$ & $P_{f a}$ & 0 & 0 \\
& $w_{a}$ & $P_{a f}$ & 0 & $P_{a w}$ & 0 \\
& $w_{w}$ & 0 & $P_{w a}$ & 0 & $P_{w r}$ \\
& $w_{r}$ & 0 & 0 & $P_{w r}$ & 0 \\
\hline \multirow{3}{*}{ LCOM } & $w_{f}$ & 0 & $P_{f a}$ & $P_{f w}$ & $P_{f r}$ \\
& $w_{a}$ & $P_{a f}$ & 0 & $P_{a r}$ & $P_{a r}$ \\
& $w_{w}$ & $P_{w f}$ & $P_{w a}$ & 0 & $P_{w r}$ \\
& $w_{r}$ & $P_{r f}$ & $P_{r a}$ & $P_{r w}$ & 0 \\
\hline
\end{tabular}

Note: $P_{j k}$ represents the connection probability from $j$ to $k ; \Sigma P_{j k}=1$. SDOM: Small Decentralized Operation Mode; LCOM: Large Centralized Operation Mode; $w_{f}, w_{a}, w_{w}$, and $w_{r}$ represents different categories of participants, including farmers, assemblers, processors and wholesalers, retailers, respectively.

Probability (Table 1) is set under the following consideration:

First, in many developing countries (including most of the region), like China, where small-scale producers have low output, limited trading skills, lack of basic transportation facilities, low education, and isolated geographical conditions, such as Ethiopia's grain $^{[25]}$ market and Philippines rice products ${ }^{[23]}$. In these economies, direct trading between wholesalers and producers is mostly absent, so assembler exercises a coordination role facilitating product transmission, the process of gathering and collecting products is an important link affecting the effective establishment of sales networks. Small-scale producers and intermediaries' cooperation mode is the best choice to reduce transaction costs and improve transaction efficiency ${ }^{[2,25]}$.

Second, farmers and rural market (Da Ji) is common in many counties in rural areas in China ${ }^{[26]}$, where farmers can sell agricultural products to other farmers or local residents on a regular date (such as every sixth day of each lunar month), its trading probability $\left(P_{f f}\right)$ is denoted in Table 1 . Similarly, the trading probabilities of the farmers' products to assemblers and wholesalers are denoted by $P_{f a}$ and $P_{f w}$, respectively.

Third, many previous models assume that the nodes are not allowed to trade cross-level, namely each level of the node must be provided by the node at the next higher level ${ }^{[27,28]}$.

As recreated in the SDOM scenario (Figure 2), a wholesaler is supplied exclusively by intermediaries, while an intermediary is only supplied by producers. These restrictions determine a specific supply network structure, where every firm is supplied only by firms in the precedent level. While under the LCOM scenario, nodes can trade across layers and establish links, and the optional range of connections at the local world of the first level also increases accordingly, which greatly expands the range of their options.

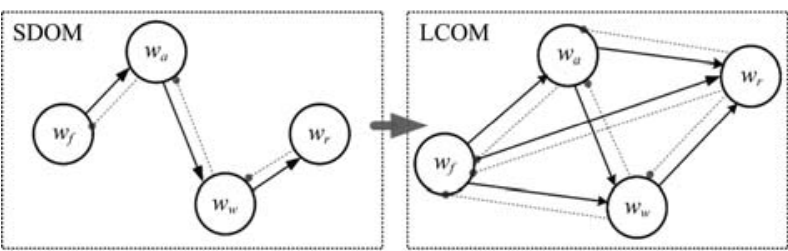

Note: The solid line arrow represents supply, and the dotted line represents procurement.

Figure 2 Local environment connection diagram of the first layer of agricultural product supply chain

2) Second level local world

After a new node is selected by its first level of local world, they then are selected by its second level local world. BA-Model's "preference growth" makes the "older" node in the network are more likely to become the "core" node, while
BB-Model through fitness in describing the connection probability ${ }^{[16]}$. Knoar et al. ${ }^{[29]}$ used gravitational mechanism to simulate the selection process of node edges, and local environment.

To assume that three influence factors are affecting its connection. The new node's product competitiveness, as well as the perishability of the product, are the current state of the nodes in the network. A node in this local-world is chosen with probability given by Equation (1).

$$
\prod_{\text {local }}(j \rightarrow i)=r \prod_{\text {status }}+q \prod_{\text {weight }}+u \prod_{\text {perish }}
$$

where, $j$ stands for the newly added node; $i$ stands for the node to be connected; $r, q$, and $u$ denote the current state of the node in the network, the product competitiveness of the newly added node, and the freshness of the product, respectively, in a certain unit of time, $r+q+u=1 . \quad \prod_{\text {local }}, \prod_{\text {status }}, \prod_{\text {weight }}, \prod_{\text {perish }}$ denote the linking probability discussed as follow:

\section{3) Status of current nodes}

The current state of the node is represented by node degree. A new node is connected to $e_{1}$ nodes in the selected local world by taking the current degree value of the node as the priority selection mechanism. The connection probability distribution follows Equation (2).

$$
\prod_{\text {status }}(j \rightarrow i)=\frac{k_{i}+\alpha}{\sum_{j \in \mathrm{LW}}\left(k_{j}+\alpha\right)}
$$

where, $k_{i}$ represents the degree of node $i$ in the current local world LW, the variable parameter $\alpha>0 \#$ is mainly used to ensure that isolated nodes can also be connected to an edge.

4) Competitive power and weight

$$
\prod_{\text {weight }}(j \rightarrow i)=\frac{\eta_{j} S_{j}}{\sum_{l \in \mathrm{LW}} \eta_{l} S_{l}}
$$

where, $\eta_{j}$ denotes the competitive power of a node; $S_{j}$ indicates the maximum volume of product that the node can produce or distribute.

5) Perishability

$$
\prod_{\text {perish }}(j \rightarrow i)= \begin{cases}1, & t<\text { Perish days } \\ 0, & t \geq \text { Perish days }\end{cases}
$$

To sum up, the connection probability of the second level local world follows Equation (5):

$$
\prod_{\text {local }}(j \rightarrow i)=r \frac{k_{i}+\alpha}{\sum_{j \in \mathrm{LW}}\left(k_{j}+\alpha\right)}+q \frac{\eta_{j} S_{j}}{\sum_{l \in \mathrm{LW}} \eta_{l} S_{l}}+u \prod_{\text {perish }}
$$

\section{3 “Asynchronous continuation" mechanism of connected edges}

This section illustrates the "retention" and "disconnection" mechanisms of connected edges. The durability of the trading relationship of fresh agricultural products is usually not high than that in other industries. The "long-term \& stable" connection between nodes will not be established after the transaction, but conditional disconnection after a certain period of continuance, which is called the "asynchronous continuation" mechanism in this study. Conditions for disconnection include:

1) Isolated nodes will disappear from the network. After a $\Delta T$ time interval, the node with no node connection is established, namely node degree $k_{i}=0$ will be forced to leave from the market competition;

2) Nodes that cannot make profits (income cannot cover its costs) will gradually disappear in the network (namely $\left.\Delta T=12\|\&\| k_{\text {in }}<k_{\text {out }}\right)$, where $k_{\text {in }}$ and $k_{\text {out }}$ represent the in-degree and 
out-degree of that node in the current local world LW, respectively. Nodes will leave the network/market after they perish also all edges to it will perish at the same time.

All connection in the simulations is zero-truncated, so every node in the network has at least one link with a firm in the upper/lower level. By means of this restriction, set aside the case of suppliers or retailers that are not integrated into the supply chain or wholesalers without relationships with either producers or retailers

The mechanism of "asynchronous continuation" is fundamentally different from the existing MLW model in the random deletion of edges, which is helpful to simulate the temporality and seasonality of fresh agricultural products supply network, avoid the generation of isolated nodes and isolated local environment, and improve the network's ability to depict real trading relationships (Figure 3 ).
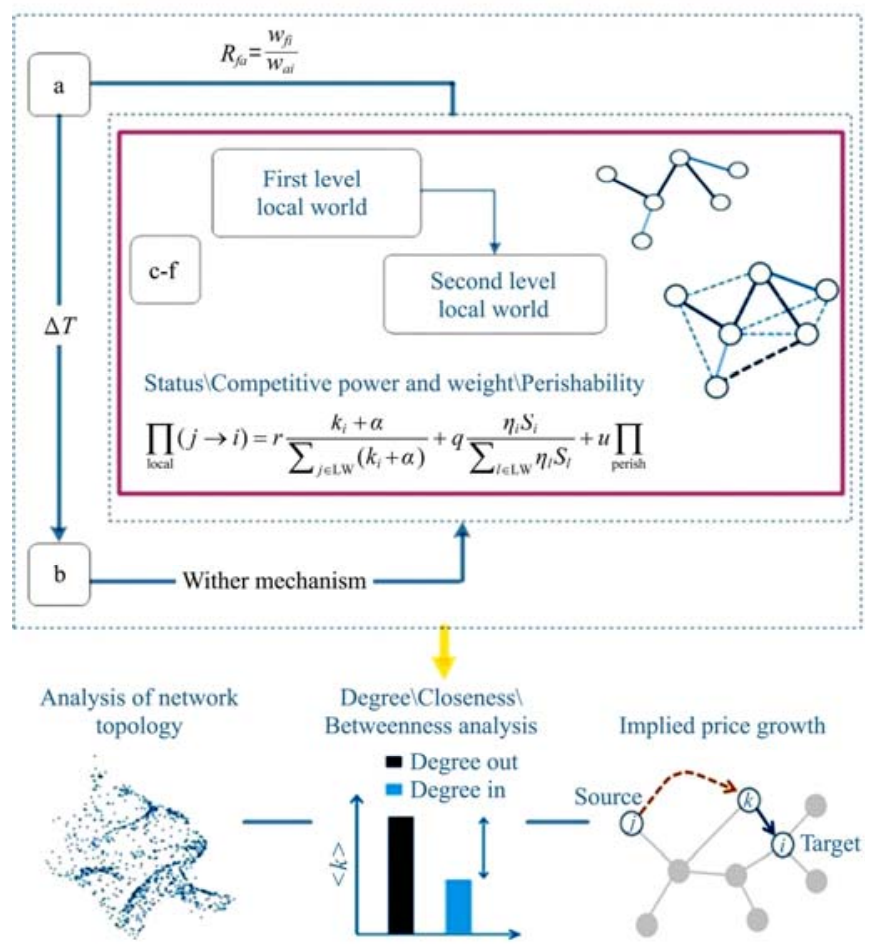

Note: Alphabets a-b denotes edge connection mechanism and c-f denotes the "Asynchronous Continuation" mechanism of connected edges.

Figure 3 Illustration of network construction and analysis structure

\subsection{Main feature of complex network models}

The characteristics of complex models can mainly be described based on the following indicators:

1) Small world feature

The path length of any two nodes is defined as the minimum number of edges connecting them in the network, Average Path Length $\bar{\ell}$ (APL) defined as the Average Path Length of all nodes, $\bar{\ell}=\frac{2}{N(N-1)} \sum_{i \geq j} d_{i j}$, where $d_{i j}$ is the shortest distance between any two nodes $i$ and $j$ in the network, $N$ is the number of nodes.

In actual social, ecological, and other networks, information transmission can be accelerated through short paths, network with many short paths are small-world featured networks, in which adjusting a small number of connections can dramatically change the performance of the network. Such as telephone networks, changing a few lines can significantly improve the performance of the system.

\section{2) Scale-free characteristics}

Most real-world networks are not randomly connected, few nodes usually have a large number of connections, while most nodes have few connections. The degree distribution of nodes conforms to a power-law distribution, which is known as the scale-free feature of networks.

Scale-free feature reflects the heterogeneity of a network, in which node connections are unevenly distributed. The previous study revealed that scale-free networks show both robustness against random failures and vulnerability to deliberate attacks ${ }^{[30,31]}$, it is possible to paralysis the whole network completely only by attacking 5\%-10\% nodes, with degrees higher than 5 . Literatures $[27,28,32]$ further studied other heterogeneous topologies of the network. The existence of power-law distribution in scale-free networks greatly improves the possibility of the existence of high-number nodes. Therefore, this kind of robustness and vulnerability has a great influence on fault tolerance and attack resistance of the network.

3) Other features

Nodes in complex networks also tend to cluster. For example, there is always a circle of acquaintances or a circle of friends in a social network where each member knows each other. The clustering degree is the degree of network collectivization, it can reflect the relationship between one group of friends and another.

The clustering coefficient of node $i$ is $C_{i}=\frac{2 n_{\text {edge }}^{(q)}}{n_{i}(n i-1)}$, in which $k_{i}$ is the degree of node $i$ in the network, $n_{i}$ is the number of neighbor nodes of the node, $n_{\text {edge }}^{(q)}$ represents the actual number of edges between the $n_{i}$ neighbor nodes, $n_{i}\left(n_{i}-1\right) / 2$ is the maximum number of edges. The Average Clustering Coefficient of the network (ACC) $C=\frac{1}{N} \sum_{i=1}^{N} C_{i}$ reflects the probability of the clustering phenomenon occurring at all nodes in the network ${ }^{[6-33]}$, that is, the degree of overlap of the circle of friends of nodes.

In general, for regular networks, the path length between any two nodes is large, but the clustering coefficient is high. For random networks, the path length between any two nodes is shorter and the clustering coefficient is lower. However, the path length between nodes of the small-world network is small and the clustering coefficient is still high. For this reason, Qian Xuesen defined complex networks as those with self-organization, self-similarity, attractors, small world, and some or all properties of scale-free networks.

The main input and output parameters of the model are listed in Table 2 .

Table 2 Parameters in Bi-Level Multi-Local-World model

\begin{tabular}{|c|c|}
\hline Name & Parameters description \\
\hline$N_{w f}, N_{w a}, N_{w w}, N_{w r}$ & The scale of farmers, assemblers, wholesalers, and retailers \\
\hline$R_{f a}=\frac{w_{f}}{w_{a}}$ & $\begin{array}{l}\text { The ratio of farmer and assemblers, } R_{a w} \text { rest be deduced by } \\
\text { analogy }\end{array}$ \\
\hline$r$ & The ratio of state of current node \\
\hline$q$ & The ratio of competitive power and weight \\
\hline$u$ & The ratio of perishability, $r+q+u=1$ \\
\hline$\eta_{j}$ & The competitive power of node $j$ \\
\hline$S_{j}$ & The weight of node $j$ \\
\hline$\Delta T$ & Network evolve time unit \\
\hline $\bar{\ell}$ & Average path length, APL \\
\hline$C$ & Average clustering coefficient \\
\hline$<k>$ & Average degree of nodes \\
\hline
\end{tabular}




\subsection{Experimental design}

In order to verify the validity of the model, this article selects cucumber and Cucurbita pepo as sample empirical products, since Cucumbers and Cucurbita pepo are both mainly domestic varieties, the export ratio is low, almost no inventory, possess obvious seasonal characteristics.

To verify the previous findings of the authors by designing a simulation experiment conceived for Empirical parameters. Through long-term monitoring, online, offline field observation, and statistical data of the Tianjin area of China, about its planting scale, price, sales channels, product flow, as well as other sources of supply and marketing information. Set each empirical parameter as follows:

1) Initializes the scale of farmer's local world $N_{w f}$ in each region

Cucumber likes warmth and is not resistant to cold. The suitable temperature for its growth is $10^{\circ} \mathrm{C}-32^{\circ} \mathrm{C}$, and it needs a large amount of water. The suitable soil and air relative humidity are both $60 \%-90 \%$. According to agricultural statistics, in 2003, 2004, 2005, and 2006, the average yield of cucumber in Tianjin was $51786 \mathrm{~kg} / \mathrm{hm}^{2}, 51855 \mathrm{~kg} / \mathrm{hm}^{2}, 45646.5 \mathrm{~kg} / \mathrm{hm}^{2}$, and $46492.5 \mathrm{~kg} / \mathrm{hm}^{2}$, respectively (Data source: National Bureau of Statistics County data and Tianjin Statistical Yearbook). In recent years, under the normal production conditions of greenhouses, the average yield is $55000 \mathrm{~kg} / \mathrm{hm}^{2}$ per season.

Cucurbita pepo has an optimal temperature of $20^{\circ} \mathrm{C}-25^{\circ} \mathrm{C}$ during the growth period, with slow growth below $15^{\circ} \mathrm{C}$ and no growth below $8^{\circ} \mathrm{C}$. According to agricultural statistics, in 2003, 2004, 2005, and 2006, the average yield of Cucurbita pepo in Tianjin was $56674.5 \mathrm{~kg} / \mathrm{hm}^{2}, 56749.5 \mathrm{~kg} / \mathrm{hm}^{2}, 49954.5 \mathrm{~kg} / \mathrm{hm}^{2}$, and $50880 \mathrm{~kg} / \mathrm{hm}^{2}$, respectively (Data source: National Bureau of Statistics county data and Tianjin Statistical Yearbook). In recent years, under the normal production conditions of greenhouses, the average yield is about $60000 \mathrm{~kg} / \mathrm{hm}^{2}$ per season.

According to the investigation and calculation by the Data of the National Bureau of Statistics, the cucumber planting area in Tianjin accounted for $9.3 \%-12.7 \%$ of the vegetable planting area, and the Cucurbita pepo planting area accounted for about 1/5 of the cucumber planting area. To sum up, the values of network nodes under the two production and operation modes are in Table 3 and Table 4.

Table 3 Cucumber SDOM scenario supply-chain network in Tianjin, 2004

\begin{tabular}{lccccccccc}
\hline \multirow{2}{*}{$\begin{array}{c}\text { Supply } \\
\text { location }\end{array}$} & \multicolumn{4}{c}{ Cucumber } & & \multicolumn{3}{c}{ Cucurbita pepo } \\
\cline { 2 - 6 } \cline { 7 - 9 } & $N_{w f}$ & $N_{w a}$ & $N_{w w}$ & $N_{w r}$ & & $N_{w f}$ & $N_{w a}$ & $N_{w w}$ & $N_{w r}$ \\
\hline Binhai & 2377 & 297 & 149 & 743 & 475 & 59 & 30 & 149 \\
Dongli & 3909 & 489 & 244 & 1221 & 782 & 98 & 49 & 244 \\
Xiqing & 6879 & 860 & 430 & 2150 & 1376 & 172 & 86 & 430 \\
Jinnan & 2841 & 355 & 178 & 888 & 568 & 71 & 36 & 178 \\
Beichen & 5182 & 648 & 324 & 1619 & 1036 & 130 & 65 & 324 \\
Wuqing & 31097 & 3887 & 1944 & 9718 & 6219 & 777 & 389 & 1944 \\
Baodi & 12786 & 1598 & 799 & 3996 & 2557 & 320 & 160 & 799 \\
Ninghe & 6787 & 848 & 424 & 2121 & 1357 & 170 & 85 & 424 \\
Jinghai & 8447 & 1056 & 528 & 2640 & 1689 & 211 & 106 & 528 \\
Jixian & 12591 & 1574 & 787 & 3935 & 2518 & 315 & 157 & 787 \\
\hline
\end{tabular}

Note: $N_{w r}=$ Acreage/Acreage|per|household, with the real value of planting area in Tianjin area in 2004 and set Acreage per household $=0.133 \mathrm{hm}^{2}$ under SDOM scenario.
Table 4 Cucumber LCOM scenario supply-chain network in Tianjin, 2018

\begin{tabular}{lccccccccc}
\hline \multirow{2}{*}{$\begin{array}{c}\text { Supply } \\
\text { location }\end{array}$} & \multicolumn{4}{c}{ Cucumber } & & \multicolumn{3}{c}{ Cucurbita pepo } \\
\cline { 2 - 6 } \cline { 6 - 9 } & $N_{w f}$ & $N_{w a}$ & $N_{w w}$ & $N_{w r}$ & & $N_{w f}$ & $N_{w a}$ & $N_{w w}$ & $N_{w r}$ \\
\hline Binhai & 174 & 11 & 7 & 121 & 69 & 4 & 3 & 48 \\
Dongli & 218 & 14 & 9 & 151 & 87 & 5 & 4 & 61 \\
Xiqing & 1050 & 66 & 44 & 729 & 420 & 26 & 17 & 292 \\
Jinnan & 125 & 8 & 5 & 87 & 50 & 3 & 2 & 35 \\
Beichen & 480 & 30 & 20 & 333 & 192 & 12 & 8 & 133 \\
Wuqing & 2197 & 137 & 92 & 1525 & 879 & 55 & 37 & 610 \\
Baodi & 985 & 62 & 41 & 684 & 394 & 25 & 16 & 274 \\
Ninghe & 816 & 51 & 34 & 566 & 326 & 20 & 14 & 227 \\
Jinghai & 749 & 47 & 31 & 520 & 300 & 19 & 12 & 208 \\
Jixian & 622 & 39 & 26 & 432 & 249 & 16 & 10 & 173 \\
\hline
\end{tabular}

Note: $N_{w f}=$ Acreage/Acreage|per|household, with the forecasted value of planting area in Tianjin in 2018, set Acreage|per|household $=1.33 \mathrm{hm}^{2}$ under LCOM scenario.

2) Initializes the scale of other participants $N_{w a}, N_{w w}, N_{w r}$

Initialize the size $N_{w a}, N_{w w}, N_{w r}$ of the assembler, wholesaler, and retailer's local environment. Values of this study are set as $R_{f a}=8, R_{a w}=2, R_{w r}=0.20$ in SDOM scenario, set $R_{f a}=16, R_{a w}=1.5$, $R_{w r}=0.06$ in LCOM scenario. Assume that the cucumber and Cucurbita pepo production and management mode in Tianjin gradually evolves from SSOM to LCOM from 2004 to 2018.

$3)$ Initialize the proportion of factors in the current state of nodes $r$, product competitiveness $q$ and freshness preservation $u$. For simplicity, set $r=0.3, q=0.3, u=0.4$, respectively, and $r+q+u=1$.

4) Initialize $\eta_{j}$ and $S_{j}$. Assuming that $\eta_{j}$ and $S_{j}$ follow uniform, exponential and normal distribution respectively under the SDOM scenario while following power-law distribution under the LCOM scenario.

5) Assuming that the SDOM scenario was adopted mostly in 2004, and the LCOM scenario gradually was adopted in 2018.

\section{Results}

Results of the scale of nodes in 2004 and 2018 are in Table 4 and Table 5 .

Table 5 Scenario simulation results under the setting of the experiment of this study

\begin{tabular}{|c|c|c|c|c|c|c|c|}
\hline Year & Product & Model & Nodes & Edges & $\bar{\ell}$ & C & $<k>$ \\
\hline \multirow{8}{*}{2004} & \multirow{4}{*}{ Cucumber } & BI-MLW & \multirow{4}{*}{139344} & 3594130 & 5.700 & 0.0013 & 3.2 \\
\hline & & ER-Model & & 78225044 & 2.823 & 0.0370 & 1503.0 \\
\hline & & BA-Model & & 3845504 & 3.524 & 0.0047 & 3.8 \\
\hline & & BB-Model & & 3827530 & 3.110 & 0.0364 & 3.3 \\
\hline & \multirow{4}{*}{$\begin{array}{c}\text { Cucurbita } \\
\text { pepo }\end{array}$} & BI-MLW & \multirow{4}{*}{27869} & 2452940 & 5.500 & 0.0010 & 3.3 \\
\hline & & ER-Model & & 11649249 & 2.670 & 0.0310 & 836.0 \\
\hline & & BA-Model & & 139320 & 3.951 & 0.0030 & 3.9 \\
\hline & & BB-Model & & 139180 & 3.491 & 0.0169 & 3.4 \\
\hline \multirow{8}{*}{2018} & \multirow{4}{*}{ Cucumber } & BI-MLW & \multirow{4}{*}{13335} & 57091 & 4.210 & 0.0260 & 4.8 \\
\hline & & ER-Model & & 2667156 & 3.664 & 0.0291 & 400.0 \\
\hline & & BA-Model & & 66650 & 3.746 & 0.0053 & 3.9 \\
\hline & & BB-Model & & 66310 & 3.266 & 0.0306 & 3.4 \\
\hline & \multirow{4}{*}{$\begin{array}{c}\text { Cucurbita } \\
\text { pepo }\end{array}$} & BI-MLW & & 24552 & 4.400 & 0.0661 & 4.6 \\
\hline & & ER-Model & \multirow{3}{*}{5334} & 426519 & 1.978 & 0.0300 & 159.9 \\
\hline & & BA-Model & & 26645 & 3.476 & 0.0117 & 3.6 \\
\hline & & BB-Model & & 26515 & 3.021 & 0.0566 & 3.5 \\
\hline
\end{tabular}

Note: Nodes denote the number of nodes; Edges denote total edges of the network; $\bar{\ell}$ Denotes Average Path Length, APL, $\bar{\ell}=\frac{2}{N(N-1)} \sum_{i \geq j} d_{i j}, d_{i j}$ is the shortest distance between node $i$ and $j$; $C$ denotes average clustering coefficient, $C=\frac{1}{N} \sum_{i=1}^{N} C_{i}, \quad n_{\text {edge }}^{(q)}$ is the number of edges in $n_{i}$ neighbors; $<k>$ denotes average degree, $\quad<k>=\frac{1}{N} \sum_{i=1}^{N} k_{i}$. 


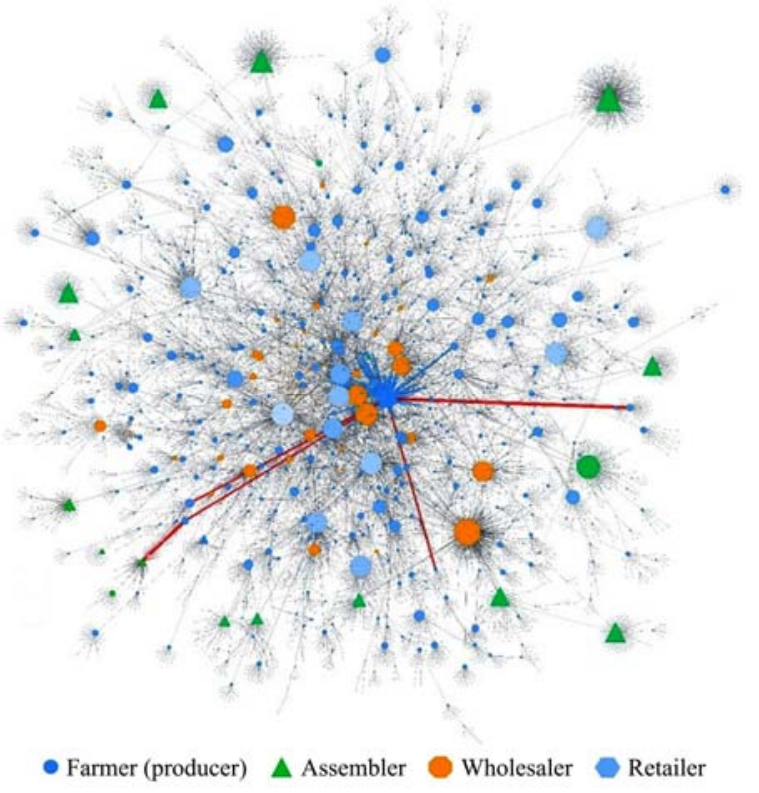

Figure 4 BI-MLW network under the SDOM Scenario of major cucumber producing areas in Tianjin, 2004

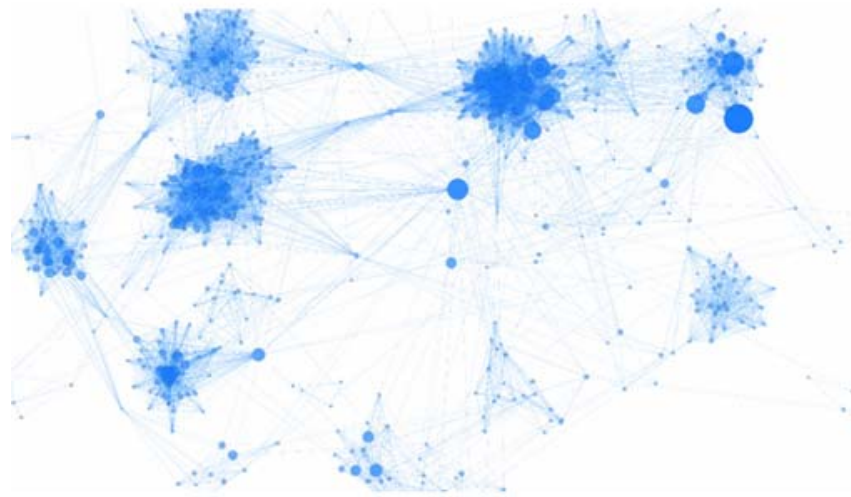

Figure 5 BI-MLW simulation Model under LCOM Scenario in cucumber production areas in Tianjin, 2018 (partial: screening with node degree greater than 30$)$

\section{Discussion}

This section attempted to evaluate the performance of the BI-MLW model from four perspectives: an overview of the network structure, node perspective of its degrees, nodes view of its average path length, and network clustering view of its average clustering coefficient. Empirical simulation and comparison are listed in Table 5.

\subsection{Network structure}

Many previous theoretical and empirical contributions proved that most real networks reflect the heterogeneity of a network, node connections are unevenly distributed with only a small number of nodes having a large number of edges, which is known as the scale-free feature of a network. Simulation of BI-MLW model also presents scale-free feature as BA, ER, and BB models. Suppose the size of circles represents the number of its connections (Figure 6). In BA and BB model, few nodes hold a majority of links, showing "the rich get richer". While in reality in some regions and seasons, farmers cannot accumulate its advantages in this manner, many farmers only sell their products to a few buyers. Hence BI-MLW model improves node ability with an "Asynchronous Continuation" mechanism discussed in Section 2.3, the degree of node will not grow unreasonably "richer" (as red dots in Figure 6), reflects the reality that even large enterprises in the field of agricultural products supply cannot occupy all markets over a short time.

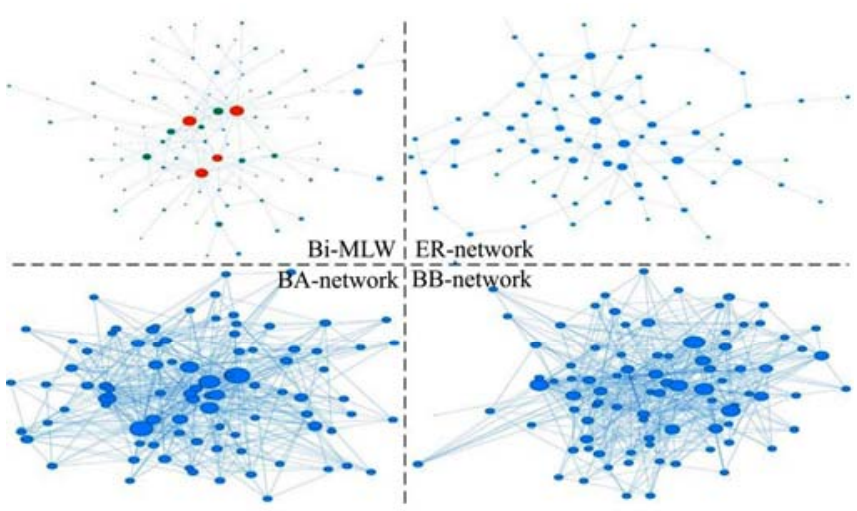

Figure 6 Comparison of Cucurbita pepo simulation model in Jinnan District, Tianjin, China in 2018

\subsection{Degree of network nodes}

Degree $k$ of $i$ represents the number of nodes connected to node $i$, reflecting the nature of each node itself. Intuitively, the larger degree indicates the higher importance of that node among all nodes. Different degree distributions can be used to describe the chain, star, or satellite network structures formed between network nodes ${ }^{[27,28,34]}$ and also describe some less common topologies such as block diagonal patterns. The average degree of the network $\left\langle k>\right.$ is the average degree of all nodes $\left\langle k>=\frac{1}{N} \sum_{i=1}^{N} k_{i}\right.$

In general, the feature of degree distribution denotes the overall properties of a network, such as fast diffusion and high destruction resistance $^{[8]}$.

As in Table 5, in 2004 average degree $<k>$ of Cucumber dealers were $3.2,1503.0,3.8$ and 3.3, which increased to 4.8, 400.0, 3.9 and 3.4 respectively in 2018. Means in 2004 average cucumber dealer only deal with 3.2 buyers, in 2018 trade with 4.8 on average, one can observe that a higher $\langle k>$ indicates a stronger ability to participate in market competition, which will reduce the heterogeneity of the network. The simulation results of the model are consistent with conclusions in other industries ${ }^{[35]}$.

\subsection{Average path length of the network}

APL $\bar{\ell}$ can reveal the average shortest path between any two nodes in the network. $\bar{\ell}=\infty$ between disconnect nodes and $\bar{\ell}=1$ in a complete connection diagram. In supply chain networks, attention should be paid to the average of the shortest length of the path from farmers to retailers through intermediaries ${ }^{[36]}$. Many actual and random networks have been found to have a small-world network characteristic ${ }^{[33,34]}$. Milgram $^{[37]}$ discovered the phenomenon of "six degrees of separation", which proves the widespread existence of small-world characteristics. Networks with small-world network characteristics, even sparse networks, all have small average shortest path length. In general, if the average path length is proportional to the logarithm of the network size $\mathrm{N}^{[38]}$, can be considered that the network is small-world characterized ${ }^{[39]}$.

Intuitively, $\bar{\ell}$ will raise as the network scales up, since products will pass through more hands with more dealers. On the contrary, simulation results of BI-MLW model (Table 5) show that $\bar{\ell}$ does not vary greatly with network size, it stays at 5.5-5.7 in 2004 and reduced to 4.2-4.4 in 2018 which fits Milgram's "six degrees theory". In real mature supply chains as General Mills, Kellogg's, Mondelez whose $\bar{\ell}$ were $3.548,3.625$, and $4.323^{[40]}$. 


\subsection{Average clustering coefficient}

The clustering coefficient represents the clustering situation near one node, large values imply a high concentration of flow in a few nodes ${ }^{[5,33]}$. high clustering facilitates coordination among firms and thereby agility hence clustering is a sign of resilience in a network

In SDOM Scenario in 2004, the Average Clustering Coefficient (ACC) $C$ of farmers' market is relatively high, while that of the whole network is not which is 0.0013 in Cucumber market and 0.0010 in Cucurbita pepo market. Under LCOM scenario in 2018, $C$ increases to 0.0260 in Cucumber market and 0.0661 in Cucurbita pepo market. This shows that under SDOM farmers mainly sell their agricultural products by means of self-supply in small areas, such as village and town fairs. On the contrary, under LCOM farmers expanded their market, hence increased the average clustering coefficient of the network.

According to the previous analysis, a moderately decentralized network structure, relatively high average degree, roughly constant average path length, a growing cluster coefficient is preferable for food supply chains, where restricted relationship SCs are common. A comprehensive comparison of these properties indicates that BI-MLW model established in this study can improve the descriptive ability as network structure, node degree, nodes average path length, and network clustering than ER, BA, BB network models in simulating the variation of fresh agricultural products supply chain.

\section{Conclusions and implications}

A complex network model between participants in the supply network under SDOM and LCOM scenarios was discussed in this study. The results showed that:

1) Network topology and its trading capacity vary under different operation scenarios. First, production scale, standardized production capacity, and quality control capacity will bring great uncertainty to the yield and quality, production has obvious seasonality, in SDOM scenario, the supply network shows generalized scale-free characteristics, with large average path length, small network clustering coefficient, few clustering cooperation between nodes, and low network efficiency and stability. Second, in the LCOM scenario, the average path length becomes shorter and the clustering coefficient becomes larger.

2) The stability of the connection relationship is low, which requires the local environment size to be highly matched. Due to the uneven quality specifications and quality of primary fresh agricultural products, the small value of single products, and the low stability of producers, the transaction links have a high degree of promptness, namely, the occurrence and instability. In addition, the freshness will decline continuously and irreversibly since the harvest, and the value will decline at different rates with time. The operators need to complete the processes of classification, primary processing, packaging, and transportation within a limited time. The simulation results also show that the unstable connection relationship requires a higher matching degree among local worlds, otherwise, it is easy to cause the failure of the connection and thus lead to the failure of the sales channel.

3) The heterogeneity of nodes in the network will not increase rapidly, and the cumulative advantage of nodes cannot be accumulated permanently. The model verifies nodes with a high degree will not significantly increase the number of edges along with the evolution of the network, i.e., the heterogeneity of the network is less prominent than that of the conventional network model, which proves that the BI-MLW model established in this study, to a certain extent, can simulate the feature that nodes with high degree value do not increase edges more rapidly. It better reflects the characteristics that even large firms in the field of agricultural products supply cannot occupy all markets quickly, but fluctuate back and forth with seasons and products.

Compared with other research, the following aspects are explored and innovated: Firstly, node types in the classical model are the same, the BI-MLW model endows different types of nodes with unique attributes and behavioral characteristics. Secondly, the random transaction of nodes in the classical model is inconsistent with reality. BI-MLW model fully considers the interaction between participants in the agricultural supply chain in reality and improves the model hypothesis in many ways. Third, the classical model is synchronous, nodes and edges exist simultaneously or die synchronously. BI-MLW innovatively put asynchronous mechanisms on the network, which means the establishment and die are closely related to the actual transaction process. Empirical results show that improvements in BI-MLW model to some extent reduced the uncertainty during the network evolvement and improved the modeling performance of the whole system.

This study can be extended in several ways. Fresh agricultural products supply chain network is highly susceptible to fluctuations as production mode, matching degree of different factors, and unique product perishability features. To that end, seeking even accurate numerical expressions to describe dynamics of nodes and edges, improving parameters of simulation scenarios through vast real-world data validation are still to be analyzed.

\section{Acknowledgements}

The authors would like to acknowledge the support of Technology Innovation Project Fund of the Chinese Academy of Agricultural Sciences (CAAS-ASTIP-2020-AII-01); the Agricultural Monitoring and Early Warning Research Team of Agricultural Information Institute of Chinese Academy of Agricultural Sciences.

\section{[References]}

[1] Newman M E J, Moore C, Watts D J. Mean-field solution of the small-world network model. Physical Review Letters, 2000; 84(14): 3201-3204.

[2] Braha D, Bar-Yam Y. Information flow structure in large-scale product development organizational networks. Journal of Information Technology, 2004; 19(4): 244-253.

[3] Wang X F, Li X, Chen G R. Complex network theory and its application. Beijing: Tsinghua University Press, 2006; 260p. (in Chinese)

[4] Zhou T, Bai W J, Wang B H, Liu Z J, Yan G. A brief review of complex networks. Progress in Physics, PIP, 2005; 34: 31-36. (in Chinese)

[5] Albert R, Barabási A-L. Statistical mechanics of complex networks. Reviews of Modern Physics, 2002; 74(1): 47-97.

[6] Ren Z M, Zeng A, Zhang Y C. Structure-oriented prediction in complex networks. Physics Reports-Review Section of Physics Letters, 2018; 750: 1-51. doi:10.1016/j.physrep.2018.05.002.

[7] Newman M E J. Clustering and preferential attachment in growing networks. Physical Review E, 2001; 64(2): 025102. doi: 10.1103/PhysRevE.64.025102.

[8] Wojtowicz W M, Vielmetter J, Fernandes R A, Siepe D H, Eastman C $\mathrm{L}$, Chisholm G B, et al. A human IgSF cell-surface interactome reveals a complex network of protein-protein interactions. Cell, 2020; 182(4): 1027-1043.

[9] Garlaschelli D, Boguñá M, Loffredo M I. The world trade web: 
Structure, evolution and modeling. UNESCO-EOLSS-Sample Chapters-Technology, Information and Systems Management Resources-Complex Networks, 2010. Available at: http://www.eolss.net/sample-chapters/C15/E6-200-05.pdf. Accessed on [2020-01-20].

[10] Wang B H, Zhou T, Zhou C S. Statistical physics research for human behaviors, complex networks, and information mining. Journal of University of Shanghai for Science and Technology, 2012; 34(2): 103-117. (in Chinese)

[11] Hofman J M, Sharma A, Watts D J. Prediction and explanation in social systems. Science, 2017; 355(6234): 486-488.

[12] Erdös P, Rényi A. On random graphs. Publicationes Mathematicae, 1959; 6: 290-297.

[13] Barabási A-L, Albert R. Emergence of scaling in random networks. Science, 1999; 286(5439): 509-512.

[14] Garlaschelli D, Loffredo M I. Structure and evolution of the world trade network. Physica a-Statistical Mechanics and its Applications, 2005; 355(1): 138-144.

[15] Li X, Jin Y Y, Chen G R. Complexity and synchronization of the world trade web. Physica a-Statistical Mechanics And Its Applications, 2003; 328(1-2): 287-296.

[16] Bianconi G, Barabási A-L. Competition and multiscaling in evolving networks. Europhysics Letters, 2001; 54(4): 436-442.

[17] Caldarelli G, Marchetti R, Pietronero L. The fractal properties of Internet. Europhysics Letters, 2000; 52(4): 386-391.

[18] Chen G R. Problems and challenges in control theory under complex dynamical network environments. Acta Automatica Sinica, 2013; 39(4): 312-321. (in Chinese)

[19] Fan Z, Chen G, Zhang Y. A comprehensive multi-local-world model for complex networks. Physics Letters A, 2009; 373(18-19): 1601-1605.

[20] Virkar Y, Clauset A. Power-law distributions in binned empirical data. Annals of Applied Statistics, 2014; 8(1): 89-119.

[21] Beamon B M. Supply chain design and analysis: Models and methods. International Journal of Production Economics, 1998; 55(3): 281-294.

[22] Kim S H, Yoon S-G, Chae S H, Park S. Economic and environmental optimization of a multi-site utility network for an industrial complex. Journal of Environmental Management, 2010; 91(3): 690-705.

[23] Hayami Y, Kikuchi M, Marciano E B. Middlemen and peasants in rice marketing in the Philippines. Agricultural Economics, 1999; 20(2): 79-93.

[24] Arya A, Loeftier C, Mittendorf B, Pfeiffer T. The middleman as a panacea for supply chain coordination problems. European Journal of Operational Research, 2015; 240(2): 393-400.

[25] Gabre-Madhin E Z. The role of intermediaries in enhancing market efficiency in the Ethiopian grain market. Agricultural Economics,
$2001 ; 25(2-3): 311-320$

[26] Wang B, Yu S H. Report on The development of China's agricultural product circulation. China Business and Market, 2009; 1: 13-17. (in Chinese)

[27] Capaldo A, Giannoccaro I. How does trust affect performance in the supply chain? The moderating role of interdependence. International Journal of Production Economics, 2015; 166: 36-49.

[28] Kim Y, Chen Y-S, Linderman K. Supply network disruption and resilience: A network structural perspective. Journal of Operations Management, 2015; 33-34(1): 43-59.

[29] Konar M, Dalin C, Suweis S, Hanasaki N, Rinaldo A, Rodriguez-Iturbe I. Water for food: The global virtual water trade network. Water Resources Research, 2011; 47(5): W05520. doi: 10.1029/2010WR010307.

[30] Nair A, Vidal J M. Supply network topology and robustness against disruptions - an investigation using multi-agent model. International Journal of Production Research, 2011; 49(5): 1391-1404.

[31] Zhao K, Kumar A, Yen J. Achieving high robustness in supply distribution networks by rewiring. IEEE Transactions on Engineering Management, 2011; 58(2): 347-362.

[32] Xu G, Feng J, Chen F, Wang H, Wang Z. Simulation-based optimization of control policy on multi-echelon inventory system for fresh agricultural products. Int J Agric \& Biol Eng, 2019; 12(2): 184-194.

[33] Newman M E J. Random graphs as models of networks. Random Graphs as Models of Networks, 2002; pp.35-68.

[34] Barrat A, Weigt M. On the properties of small-world network models. European Physical Journal B, 2000; 13(3): 547-560.

[35] Kim Y, Choi T Y, Yan T, Dooley K. Structural investigation of supply networks: A social network analysis approach. Journal of Operations Management, 2011; 29(3): 194-211.

[36] Dorogovtsev S N, Mendes J F F, Samukhin A N. Structure of growing networks with preferential linking. Physical Review Letters, 2000; 85(21): 4633-4636.

[37] Milgram S. The small world problem. Psychology Today, 1967; 2: $60-67$.

[38] Albert R, Jeong H, Barabási A-L. Diameter of the world-wide web. Nature, 1999; 401(6749): 130-131.

[39] Travers J, Milgram S. An experimental study of the small world problem. Sociometry, 1969; 32: 425-443.

[40] Orenstein P. How does supply network evolution and its topological structure impact supply chain performance? 2016 Second International Symposium on Stochastic Models in Reliability Engineering, Life Science and Operations Management (SMRLO). IEEE, 2016; pp.562-569. 